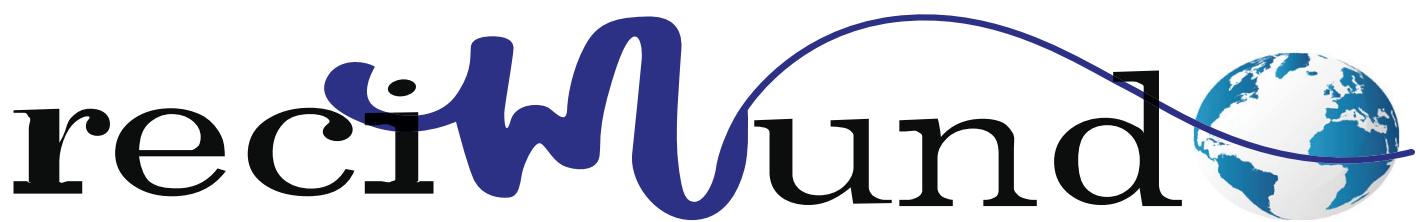

Revista Científica Mundo de la Investigación y el Conocimiento

DOI: 10.26820/recimundo/5.(Suple1).oct.2021.149-163

URL: https://recimundo.com/index.php/es/article/view/1336

EDITORIAL: Saberes del Conocimiento; Universidad Yachay Tech

REVISTA: RECIMUNDO

ISSN: 2588-073X

TIPO DE INVESTIGACIÓN: Artículo de investigación

CóDIGo UNESCO: 32 Ciencias Médicas

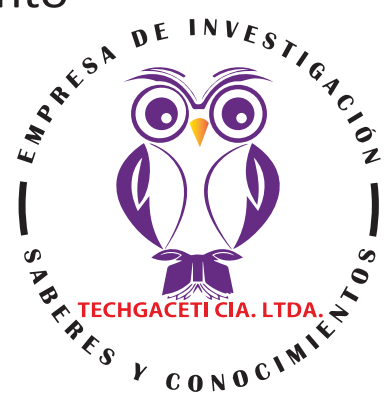

PAGINAS: $149-163$

\title{
La Farmacología en tiempos de COVID-19: Desafíos de su enseñanza y aplicación profesional
}

\section{Pharmacology in times of COVID-19: Challenges of its teaching and professional application}

La Farmacología en tiempos de COVID-19: Desafíos de su enseñanza y aplicación profesional

\section{Jhon Alexander Ponce Alencastro ${ }^{1}$}

RECIBIDO: 02/09/2021 ACEPTADO: 20/09/2021 PUBLICADO: 30/10/2021

1. Doctor en Medicina y Cirugía; Especialista en Atención Primaria de la Salud; Magíster en Docencia e Investigación Educativa; Posgrado en Diabetología y Geriatría; Docente Investigador de la Universidad Técnica de Manabí; Portoviejo, Ecuador; Jhon.ponce@utm.edu.ec; iD https://orcid.org/0000-0002-3666-7865

CORRESPONDENCIA

Jhon Alexander Ponce Alencastro

jhon.ponce@utm.edu.ec.

Quito, Ecuador

() RECIMUNDO; Editorial Saberes del Conocimiento, 2021 


\section{RESUMEN}

Introducción: La educación médica ha tenido cambios radicales durante los últimos cincuenta años, gracias a los avances en el campo de la genética y la biomedicina, sin dejar de lado los grandes aportes de la Farmacología. Todo lo anterior ha tenido otro gran impulso frente a la pandemia por Covid - 19 que ha puesto en el borde de lo conocido y lo desconocido en el manejo de enfermedades infecciosas y su repercusión en las características biológicas, psicológicas y sociales del ser humano. Objetivo: El objetivo central de este artículo es identificar los desafíos de la enseñanza de la Farmacología y su aplicación, en el contexto de la pandemia por COVID-19 en la elaboración de una fuente de información académicamente relevante, global y justificable. Método: Se trata de una investigación documental ubicada en el paradigma postpositivista con un enfoque cualitativo, partiendo de la búsqueda, recopilación y revisión de fuentes bibliográficas recuperadas de bases de datos de acceso libre como PubMed, Dialnet, Redalyc y Scielo en inglés y español. Resultado: Se logró identificar los desafíos que plantea la enseñanza y la aplicación de la Farmacología a través de un documento diseñado con la revisión total de 35 artículos científicos de acceso libre y gratuito incluyendo textos físicos y digitales. Conclusión: La Farmacología es una disciplina indispensable en la formación del profesional de la salud para mejorar la toma de decisiones, las habilidades clínicas y disminuir el error médico en el abordaje terapéutico de las enfermedades como el caso de la Covid - 19.

Palabras clave: Farmacología; Covid-19; Educación médica; Tratamiento.

\section{ABSTRACT}

Introduction: Medical education has undergone radical changes during the last fifty years, thanks to advances in the field of genetics and biomedicine, without neglecting the great contributions of Pharmacology. All of the above has had another great boost in the face of the Covid-19 pandemic that has put the known and the unknown on the edge of the management of infectious diseases and its impact on the biological, psychological and social characteristics of the human being. Objective: The main objective of this article is to identify the challenges of teaching Pharmacology and its application, in the context of the COVID-19 pandemic in the development of an academically relevant, global and justifiable source of information. Method: It is a documentary research located in the postpositivist paradigm with a qualitative approach, based on the search, compilation and review of bibliographic sources recovered from open access databases such as PubMed, Dialnet, Redalyc and Scielo in English and Spanish. Result: It was possible to identify the challenges posed by the teaching and application of Pharmacology through a document designed with a total review of 35 free and open access scientific articles including physical and digital texts. Conclusion: Pharmacology is an essential discipline in the training of health professionals to improve decision-making, clinical skills and reduce medical error in the therapeutic approach to diseases such as Covid - 19.

Keywords: Pharmacology; Covid-19; Medical education; Treatment.

\section{RESUMO}

Introdução: A educação médica sofreu mudanças radicais nos últimos cinquenta anos, graças aos avanços no campo da genética e da biomedicina, sem descurar as grandes contribuições da Farmacologia. Tudo isso teve outro grande impulso em face da pandemia Covid-19, que colocou o conhecido e o desconhecido no limite da gestão de doenças infecciosas e seu impacto nas características biológicas, psicológicas e sociais do ser humano. Objetivo: o objetivo principal deste artigo é identificar os desafios do ensino da Farmacologia e sua aplicação, no contexto da pandemia COVID-19, no desenvolvimento de uma fonte de informação academicamente relevante, global e justificável. Método: trata-se de uma pesquisa documental situada no paradigma pós-positivista com abordagem qualitativa, baseada na busca, compilação e revisão de fontes bibliográficas recuperadas de bases de dados de acesso aberto como PubMed, Dialnet, Redalyc e Scielo em inglês e espanhol. Resultado: Foi possível identificar os desafios colocados pelo ensino e aplicação da Farmacologia através de um documento elaborado com uma revisão total de 35 artigos científicos de acesso livre e aberto incluindo textos físicos e digitais. Conclusão: A Farmacologia é uma disciplina essencial na formação de profissionais de saúde para melhorar a tomada de decisão, habilidades clínicas e reduzir o erro médico na abordagem terapêutica de doenças como Covid - 19.

Palavras-chave: Farmacologia; Covid19; Educação médica; Tratamento. 


\section{Introducción}

A raíz de las medidas de distanciamiento social adoptadas por la pandemia COVID-19 en diferentes áreas laborales, se dieron cambios significativos en lo cultural y socioeconómico. Agregándose a esto el cierre de los centros educativos de todos los niveles. Siendo así, que, debido a la emergencia sanitaria aproximadamente 470.5 millones de estudiantes a nivel mundial dejaron la presencialidad, lo que generó la necesidad de implementar rápidamente estrategias de enseñanza-aprendizaje en formato virtual (Failache et al., 2020) (UNESCO, 2020).

Como parte de los esfuerzos estatales a través de la normativa transitoria para el desarrollo de actividades académicas en las Instituciones de Educación Superior (IES), debido al estado de excepción decretado por la emergencia sanitaria ocasionada por la pandemia de COVID-19, se ha asegurado parcialmente los parámetros mínimos, para superar dificultades y asegurar el derecho a la educación superior de calidad en las universidades y escuelas politécnicas del Ecuador (C. E. S., 2020).

Por lo que la capacidad de implementar nuevas formas de aprendizajes mediante el uso de las tecnologías de la informática y comunicación (TIC) ha sido el pilar para atenuar la distancia física tratando de llevar una nueva normalidad.

Considerando los beneficios que estas tecnologías han demostrado en el aprendizaje, tratando de integrar en la virtualidad, los planes de estudio diseñados para la presencialidad, pasando la virtualidad de ser complementaria, a ser la principal modalidad de estudio. Evitando la posible desvinculación de los/las estudiantes, en la continuidad de sus clases, teniendo en cuenta, que, a pesar de todos los esfuerzos realizados por las instituciones educativas, las desigualdades en la población estudiantil están cada vez más presentes.
De manera que el uso de las TIC proporciona recursos, y, sobre todo, ambientes que establecen interacciones y experiencias de conectividad e interconectividad para fomentar la innovación educativa en las IES. Priorizando el aprendizaje autónomo de los estudiantes cambiando la concepción, el diseño y el desarrollo de materiales como las guías y los compendios de estudios realizados por el personal docente (C. E. S., 2020)

Lo cual no ha sido una tarea sencilla para las Facultades de Salud, en la impartición de asignaturas con contenidos teóricos y prácticos como en el caso de la Farmacología, cuya modalidad actual de estudios no presenciales, ha ido proporcionando oportunidades a la población de estudiantes de Medicina, de continuar con la formación académica, sobrellevando las evidentes brechas digitales y condiciones socioeconómicas tanto a quienes viven en áreas urbanas, urbano marginales y rurales del Ecuador.

El objetivo central de este artículo es identificar los desafíos de la enseñanza de la Farmacología y su aplicación, en el contexto de la pandemia por COVID-19 en la elaboración de una fuente de información académicamente relevante, global y justificable.

\section{Metodología}

La presente investigación documental está ubicada en el paradigma postpositivista con un enfoque cualitativo, partiendo de la descomposición del tema en descriptores de indexación en el campo biomédico, obteniéndose palabras claves como Farmacología, Covid-19, educación médica y tratamiento. Para posteriormente llevar a cabo la búsqueda, recopilación, revisión y organización bibliográfica a través del gestor Zotero de 35 fuentes bibliográficas recuperadas de bases de datos de libre acceso como PubMed, Dialnet, Redalyc y Scielo, textos físicos y digitales tanto en inglés como en

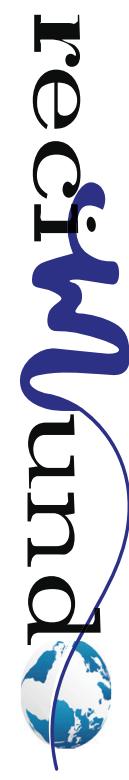


español que forman parte de la literatura médica clásica, describiendo algunas líneas teóricas y aportes al estado actual de la enseñanza de la Farmacología y su aplicación profesional, que ha tenido algunos cambios en el contexto de la pandemia por COVID-19. Fortaleciendo el aprendizaje de los/las estudiantes de Medicina en conocimientos, habilidades, actitudes y valores a través de las actividades autónomas para llegar a la reflexión sobre el propio proceso educativo. Los criterios de inclusión fueron la disponibilidad de artículos de revistas indexadas con una antigüedad no mayor a los últimos 10 años y textos tanto físicos como digitales con una antigüedad menor a 10 años, excepto aquellos contenidos que resulten relevantes y clásicos en la literatura médica que sean mayores a este período de tiempo.

\section{Desarrollo}

\section{Desafíos en la enseñanza de la Farma- cología}

La Farmacología es una ciencia que depende de otras disciplinas de la Medicina, constituyendo una especialidad sanitaria que toma de cada disciplina los elementos claves para escoger la mejor estrategia terapéutica y poder recuperar la homeostasis de un organismo amenazado por una determinada patología (Ponce, 2020).

Pero no solo para la Medicina es importante la Farmacología, sino también para otros campos de la salud como Enfermería, Nutrición, Odontología, Medicina Veterinaria, Bioquímica y Farmacia, orientándose esta relevancia en la comprensión de los efectos de la terapia medicamentosa.

Considerando a la Farmacología una disciplina, porque representa una parte del conocimiento científico, que aborda en el diseño curricular un verdadero interés académico. Pudiendo impartirse en varios niveles de estudio (semestres), que en la UTM se im- parte como Farmacología I y Farmacología II, en quinto y sexto nivel respectivamente, pudiendo abarcar varias asignaturas tanto clínicas como quirúrgicas.

Ponce (2021b) enfatiza que, la Farmacología es una disciplina integrada con los aportes de las ciencias básicas y las ciencias especializadas de la salud, cuya finalidad es conferir las herramientas necesarias para una práctica clínica más razonable y basada en evidencia.

Y a partir de estos preceptos disciplinarios, se fundamenta a la Farmacología como una asignatura teórica - práctica que ha tenido algunas innovaciones en su enseñanza los últimos años, sobre todo en la parte práctica, con la incorporación de softwares simuladores de laboratorio, así como, la revisión y discusión de estudios de casos clínicos farmacológicos.

Para Beltrán et al. (2016) la asignatura es un sistema que integra los temas y como subsistema de la disciplina expresa un ordenamiento lógico y pedagógico de contenido a ese nivel, subordinado a la disciplina. Posibilitando al estudiante caracterizar una parte de la realidad objetiva, que resuelva los problemas inherentes a ese objeto en un plano teórico, que tiene un objetivo cuya habilidad es compleja y de un orden de sistematicidad también complejo integrando en un sistema de operaciones aquellas que aparecen como habilidades a nivel de tema. Tomando en cuenta estos principios teóricos generales, la Farmacología como tal, se encasilla como una ciencia fundamental, en continuo crecimiento, cuyos intereses son afines y se enlazan con otras asignaturas básicas de la Medicina, buscando en los principios de estas asignaturas, la solución de problemas específicos que afectan el organismo, derivados de patologías prevalentes y no prevalentes (Vera, 2014).

Porque la prescripción e indicación adecuada de un medicamento, depende de un 
conocimiento farmacológico actualizado, acorde a la enfermedad y basado en evidencia científica. Pero a pesar de cumplir estos requerimientos, la respuesta farmacológica no siempre es la esperada, debido a la variabilidad en la respuesta de la gran mayoría de los medicamentos.

Siendo esta variabilidad dependiente de las alteraciones propias del individuo, de las propiedades físico - químicas medicamentosas y de las interacciones entre fármacos; ante la posible interferencia que los nutrientes hacen con la absorción, el metabolismo y la eliminación de fármacos (Ponce, 2021b).

En este sentido el tener presente los principios farmacológicos generales, las características fenotípicas de cada patología y las constantes fisiológicas del ser humano por ciclos de vida, aportan al dominio teórico práctico de la Farmacología.

Y si bien educar supone un despertar humano (Picón, 2020), la enseñanza de la Farmacología en la modalidad virtual ha tenido que hacer algunas adaptaciones, tomando en consideración los siguientes desafíos:

a. Innovación de estrategias metodológicas en las actividades de aprendizaje a la modalidad no presencial, con apoyo de las TIC, para diseñar, producir y evaluar recursos educativos, en busca de la excelencia y la motivación para el mejoramiento de la calidad de los servicios, especialmente, en el cumplimiento de la misión de la universidad a través de los planes curriculares para ejecutarlos en la interacción docente - estudiante generando un aprendizaje significativo que implique esta relación.

b. Gestión del desarrollo social y cognitivo a través del apoyo, la instrucción y la valoración a lo largo de todo el proceso educativo. Tomando como ejes de intervención las funciones sustantivas que garantizan la consecución de los fines de la educación superior; las cuales figuran como: docencia, investigación y vinculación (C.E.S, 2019).

c. Construcción de acervos del conocimiento en base a la combinación de la experiencia previa (conocimientos de ciencias preclínicas) con las nuevas informaciones (conocimientos de ciencias clínicas), transformando la realidad social que requiere del conglomerado de estudiantes conscientes, comprometidos y proactivos.

d. Fortalecimiento de la relación docente estudiante, la cual es la clave en el proceso de la formación académica para el desarrollo de competencias interpersonales, promoviendo el logro de relaciones más empáticas que generen confianza, compromiso y disciplina, a través de la adquisición de nuevos conocimientos en beneficio de la sociedad.

No obstante, de todos estos desafíos, uno de los que mayores implicaciones tiene, es la innovación de estrategias metodológicas idóneas (gráfico 1) a través de actividades favorecedoras del proceso enseñanza aprendizaje; sobre todo, cuando se aplica una metodología presencial a la no presencialidad, manteniendo la calidad sin dejar de lado el mejoramiento de la misma.

Persistiendo la negativa de ciertos docentes al uso de las TIC, resistiéndose a pesar del desarrollo tecnológico actual; marcando un nuevo paradigma de enseñanza que da lugar a nuevas metodologías y nuevos roles del docente en el cumplimiento de las actividades de aprendizaje a partir de las funciones sustantivas (Rojas et al., 2014) (C.E.S, 2019). 


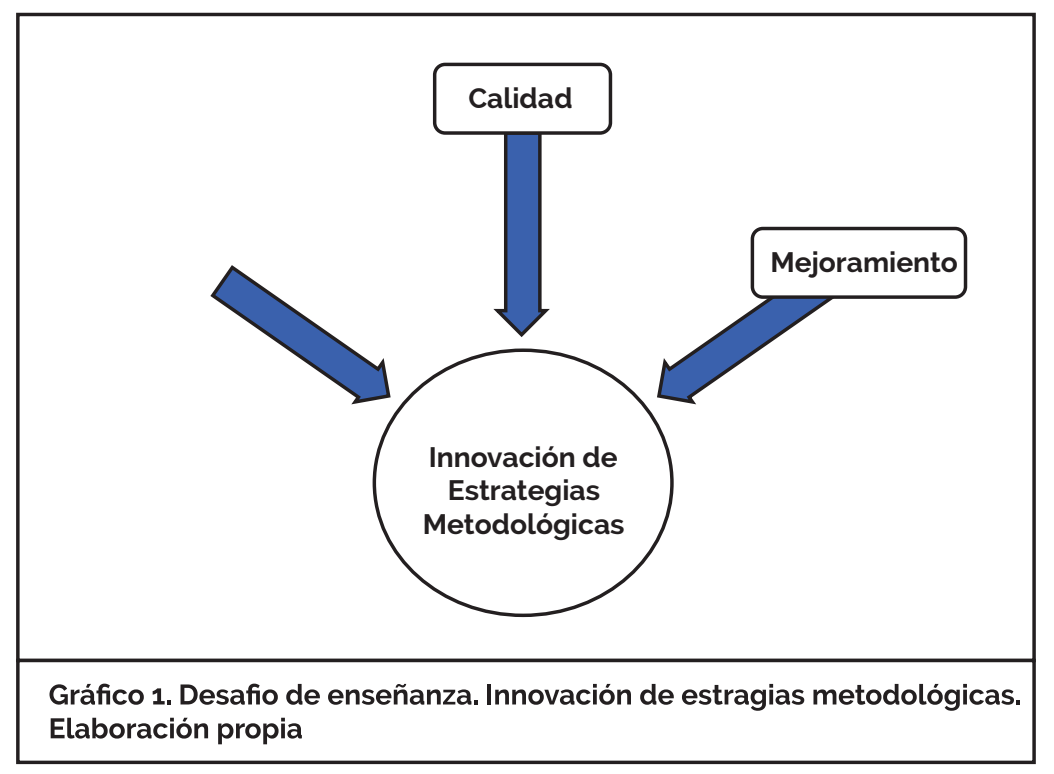

Sin que consideren muchos docentes que los conocimientos son acumulativos y con el tiempo se renuevan o cambian (Díaz, 2016). Desde luego este desplazamiento entre lo sabido y lo nuevo a saber ocurrirá ante eventos inesperados, como ha pasado con la pandemia por Covid - 19, que hacen ver el mundo de una manera más objetiva, dejando lo subjetivo en el campo de las creencias enraizadas en la resistencia al cambio organizacional.

Ante una pandemia que ha dejado al descubierto la necesidad de desarrollar una serie de conocimientos y competencias que, ante una eventual crisis, puedan continuar con sus procesos productivos; en este sentido, lo pendiente en la responsabilidad social de las IES como algo que se debe subsanar (Umaña, 2020).

Cabe resaltar que la resistencia al cambio en las instituciones educativas está inmersa dentro de los desafíos antes mencionados, manifestándose la resistencia sobre todo al uso de las TIC, obedeciendo a la falta de interés en cambiar para mejorar la institución, lo complejo que puede ser el adaptarse a lo estipulado en la normativa transitoria del CES o bien tratan de disminuir su colaboración para preservar el statu quo, llevando a cabo acciones tanto abiertas como veladas que los docentes llevan a cabo con el fin de prevenir, interrumpir o dificultar la implementación exitosa de los cambios (Córica, 2020).

Siendo muy importante, tener presente que, las TIC no son en sí mismas una innovación didáctica, sino un medio para el desempeño pedagógico. El trabajo con estas tecnologías, depende de la propuesta didáctica que las incluya, las formas del proyecto de educación a distancia, los objetivos planteados y el contexto en el que el proyecto tiene lugar (Rojas et al., 2014).

De estos desafíos se pueden tener en consideración las siguientes variantes conectadas entre sí:

- La educación no es estática ni sujeta a una sola base teórica, porque constituye un proceso dinámico y en constante evolución desde varios principios teóricos fundamentados.

- Las IES ecuatorianas Ilevan desenvolviéndose desde hace algunas décadas en la modalidad abierta y a distancia, como es el caso de la Universidad Tecnológica Particular de Loja (UTPL) que empezó por el año 1976 (Morocho y Rama, 2013), expandiéndose a los entornos virtuales con la modalidad en lí- 
NAL

nea lo que ha generado grandes efectos en la función y responsabilidad social académica; puesta a prueba en la pandemia actual.

- La educa ción superior no debe circunscribirse solo a la universidad, porque debe adaptarse a las demandas del entorno y los posibles mercados laborales, sin renunciar al espacio universitario, para promover el desarrollo de competencias, donde prime la calidad y la capacidad en el establecimiento de planes de desarrollo regional en la identificación de competencias profesionales necesarias.

\section{Regulación de la educación superior emitida por el CES en el contexto de la pandemia en el Ecuador}

El sector de la educación es uno de los más golpeados durante la pandemia, principalmente, por la transformación de las interacciones mientras se daban las adaptaciones necesarias o incluso después de ellas, lo que la situación supone desafíos globales y comunes asociados a la infraestructura tecnológica, así como, los aspectos logísticos y psicosociales (Sánchez y Ruiz, 2021)

Teniendo como uno de sus grandes retos la formación médica integral en áreas clínicas y quirúrgicas en el pregrado. La cual se desarrolla, a menudo, mediante estrategias encaminadas al desarrollo de capacidades diagnósticas, más que terapéuticas. Inclusive, es frecuente que los estudiantes copien las decisiones de prescripción de sus profesores, o las directrices terapéuticas al uso, sin que se comprendan las razones para seleccionar determinados tratamientos; creándose una costumbre de recomendar el uso de fármacos sin una base científica fundamentada.

En este aspecto, la función sustantiva de la docencia corresponde al proceso que involucra el desarrollo de capacidades y habilidades, la construcción de conocimientos, la investigación y la vinculación con la sociedad. Este se deriva de la interacción entre personal docente y estudiantes que sucede en entornos de enseñanza-aprendizaje (Sánchez y Ruiz, 2021).

Pero la capacidad de enseñanza que tengan los docentes sobre la Farmacología debe ir más allá de una actividad dinámica como es el proceso de formación de estudiantes de Medicina, más aún durante la situación de pandemia por Covid - 19, cambiando de esta manera las actividades académicas presenciales rutinarias por estrategias didácticas aplicadas en un escenario virtual.

Presentándose también ciertos avatares en este proceso de enseñanza, que a pesar de la distancia se ha ido buscando la aproximación a diversas estrategias que faciliten el aprendizaje que se pondrá posteriormente en práctica durante el ejercicio profesional.

Pero este proceso se fundamenta en el acceso a la educación, y dentro de ella la educación superior, como un derecho humano, de acuerdo a muchos tratados internacionales y sobre todo conforme lo establece la Constitución de la República del Ecuador en su artículo 26, que declara a la educación como un derecho de las personas a lo largo de su vida y un deber ineludible e inexcusable del Estado (Gob.ec, 2008).

De manera que, para alcanzar este derecho, la educación superior impartida por las universidades debe ser de calidad. Y ante esta necesidad ineludible de migrar a la modalidad en línea de la aplicación de carreras y programas diseñados para la presencialidad, se deben tener en cuenta criterios de calidad, para de esta manera, no solo garantizar el derecho a la accesibilidad por medios digitales, sino también asegurar la aceptabilidad a través de la calidad en la educación en línea (Sánchez y Ruiz, 2021). Partiendo de la planificación de los compo-

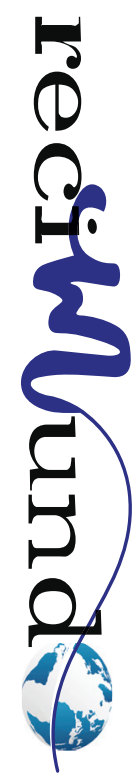


nentes teóricos de manera virtual, estableciéndose a través de las IES, Ios mecanismos para que los/las estudiantes puedan llevar a cabo el aprendizaje práctico-experimental de las carreras y programas, a través del uso de recursos y herramientas telemáticas virtuales; siempre y cuando garanticen el principio de la calidad en la educación superior y la rigurosidad académica (C. E. S., 2020).

Así mismo esta calidad también depende de las actitudes y aptitudes propicias para la utilización de las TIC en la modalidad en línea; no siendo exclusivamente la incorporación de la tecnología y su manejo instrumental lo más importante, porque hay que considerar las posibilidades de recursos y el entorno de los/las estudiantes.

Siendo importante recalcar que, a parte de la tecnología, organizar el aprendizaje autónomo en las modalidades a distancia y en línea, es una prioridad. Pero a pesar de las modificaciones que se vayan realizando por efectos de la pandemia, no se debe dejar de garantizar la calidad y la rigurosidad académica, siempre que la asignatura en cuestión lo permita (C. E. S., 2020).

A la par de superar la organización de los aprendizajes y las limitaciones en el entorno estudiantil, se va fomentando el reconocimiento que tiene el uso de las TIC en el espacio universitario, cuyo empleo permite mayor flexibilidad e interactividad; mayor vinculación con los docentes y el resto de los/las estudiantes, al permitir mayor participación y facilidad para acceder a los materiales de estudio y a otras fuentes complementarias de información (Varguillas y Bravo, 2020).

En referencia a esto la normativa transitoria del Consejo de Educación Superior (CES) menciona que, se fortalecerán los procesos de aprendizaje autónomo de los/las estudiantes, así como la ampliación, profundización y especialización de los cono- cimientos, ofreciendo al estudiante las IES, el acceso abierto al menos a una biblioteca virtual y un repositorio digital de apoyo (C. E. S., 2020).

Garantizando la continuidad de los estudios del alumnado mediante la elaboración de guías de estudio por cada asignatura, curso o su equivalente, de tal forma que el estudiante que no tenga acceso a medios tecnológicos, pueda acceder al material del curso y desarrollar las actividades académicas de la asignatura mediante el aprendizaje autónomo (C. E. S., 2020).

Y en torno al acceso a las clases impartidas, las IES que cuenten con herramientas tecnológicas que permitan la ejecución de las clases a través de medios virtuales o telemáticos de manera sincrónica entre el profesor y el estudiante, deberán contar con un repositorio que contenga las grabaciones de las sesiones de clase, de manera que los estudiantes que no pudieran asistir, tengan acceso posterior a éstas (C. E. S., 2020).

Dentro del contexto local, la UTM, ha concebido un modelo educativo presencial que se sustenta fundamentalmente en la teoría histórico - cultural de Vygotsky "el constructivismo social". Siendo este modelo universitario el que fundamenta sus bases en objetivos, políticas y estrategias que protagonicen el cambio y que contribuyan con eficiencia en el desempeño académico (Zambrano et al., 2018).

Pero debido al estado de excepción decretado por la emergencia sanitaria ocasionada por la pandemia de Covid-19 a partir de marzo de 2020, se dieron cambios de la modalidad presencial a la modalidad híbrida para dar continuidad a las actividades académicas planificadas.

Entendiéndose la modalidad híbrida como aquella que conjuga la modalidad semipresencial, en línea y a distancia, priorizando el 
aprendizaje autónomo de los/las estudiantes, para lo cual se requiere que todo curso, asignatura o su equivalente contenga una guía de estudios desarrollada por el personal académico (C. E. S., 2020).

Lo que en la mayoría de las asignaturas no se ha podido concretar debido a los ajustes y adaptaciones que se han tenido que realizar desde los planes analíticos de estudios y posteriormente en los syllabus.

Salguero (2009) y Beltrán et al. (2016) consideran a los planes analíticos como los documentos derivados de los programas de las disciplinas, que desglosan detalladamente los contenidos en temas y subtemas de cada una de las asignaturas de las diferentes áreas con el fin de precisar el desarrollo del proceso enseñanza - aprendizaje en el respectivo período académico.

Si bien su elaboración recae en la individualidad del docente, estos planes son revisados por una comisión académica que hace observaciones y/o sugerencias que deben ser consideradas en consenso con los docentes que imparten la misma asignatura, desde la experiencia docente en la UTM.

El syllabus comprende el documento, cuya principal función es la de actuar como un contrato entre el docente y el estudiante, en el que cada uno se compromete con el proceso de enseñanza - aprendizaje (Caballero, 2013).

A pesar de ya tener una organización de los documentos académicos para cada período académico, esto ha sido una tarea un poco más compleja durante la pandemia, ante una considerable población estudiantil de aproximadamente 35.228 estudiantes entre pregrado y posgrado, cuya implementación de nuevas estrategias de aprendizaje, han requerido de la organización, planificación y responsabilidad social en la pertinencia de fortalecer los campos del conocimiento en modalidad virtual, em- pleando plataformas virtuales de aprendizaje adaptables a las TIC, que involucra parámetros contextuales, cuya finalidad es garantizar la ya mencionada calidad educativa (UTM, 2019).

Empleando las plataformas virtuales, que son aplicaciones informáticas, que buscan generar comunicación académica y pedagógica entre un grupo de participantes; que puede tener un complemento mixto de tipo semipresencial y virtual, o se puede manejar de forma exclusivamente virtual (Cedeño y Murillo, 2020).

Siendo las aulas virtuales aquellos espacios dentro de las plataformas, destinados para la publicación de contenidos, que permitan el desarrollo interactivo de la docencia, con una buena integración de recursos de enseñanza aprendizaje (Rojas et al., 2014).

En la experiencia docente llevada a cabo en la UTM durante la pandemia, se ha tratado de garantizar la adquisición de las competencias y los resultados de aprendizaje de la modalidad presencial a la no presencial, partiendo de una mayor implementación de la plataforma E - virtual ante la acelerada migración virtual por la pandemia. Siendo elemental, la idónea comunicación de los directivos con el personal docente, y de estos últimos con los estudiantes, sobre los cambios que se han elaborado en los aspectos metodológicos, didácticos y la evaluación de los aprendizajes.

De acuerdo a Ponce y Salazar (2021) la extensión del aula de clases a los hogares no reemplaza el aula física, porque podemos llevar el entorno de aprendizaje, más no la esencia del aula, donde se gesta la relación docente - estudiante, en una comunidad más activa en contacto con el lenguaje corporal de cada uno, que se ve limitada por el uso exclusivo de una pantalla, tornándose una situación de gran complejidad que favorece el distanciamiento entre los pares de ambos, por más que se intente reinventar

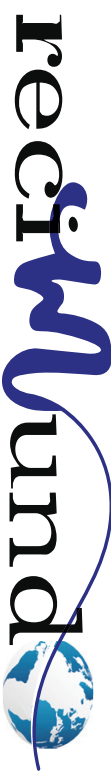


las prácticas universitarias desde una pedagogía digitalizada.

En definitiva, la pandemia nos ha limitado a permanecer en los hogares, extendiendo el aula física, sin que dejemos de tener en cuenta que se trata de un espacio virtual en una dimensión socioemocional y con un formato diferente (Picón, 2020).

\section{La enseñanza de la educación médica a través de las plataformas digitales}

La educación médica (EM) ha tenido cambios radicales durante los últimos cincuenta años, gracias a los avances en el campo de la genética y la biomedicina, sin dejar de lado los grandes aportes de la Farmacología. Cuyo enfoque clínico tanto teórico como práctico en los nuevos escenarios virtuales adoptados por la UTM presentan adaptaciones de acuerdo a la normativa transitoria vigente del CES, tratando de conservar el mismo rigor académico que la modalidad presencial.

Adoptando la necesidad docente de implementar las plataformas de gestión de aprendizaje, las cuales son herramientas para la creación de entornos de enseñanza aprendizaje, integrando materiales didácticos y herramientas de comunicación, colaboración y gestión de la educación. Donde el reto en sí no es del todo tecnológico, pues en esencia se trata de un cambio paradigmático del propio proceso docente educativo, en una sociedad donde las nuevas relaciones espacio-tiempo están modificando la comunicación humana (Rojas et al., 2014)

Considerando a la virtualización de la enseñanza como uno de los principales objetivos en la Agenda 2030 para la educación y su desarrollo implica cambios de paradigmas hacia un proceso de formación y gestión universitaria sustentado en el uso de las TIC (García et al., 2021).
En esta línea del pensamiento Orellano (2012), refiere que es necesario tener en cuenta que las tecnologías no son en sí mismas una innovación didáctica, sino un medio para el desempeño pedagógico. Cuyo trabajo, depende de la propuesta didáctica que las incluya, las formas del proyecto de educación a distancia, los objetivos planteados, el contexto en el que el proyecto tiene lugar y el rigor académico que exige.

Teniendo en cuenta que el personal docente de la UTM ha sido capacitado en el manejo de las herramientas tecnológicas del e - learning, creando aulas virtuales en donde ponen a disposición de los/las estudiantes el material del curso, la bibliografía, los recursos evaluativos en función de los resultados de aprendizaje específicos de la asignatura, en un ambiente de intercambio interactivo individual y colectivo (Rojas et al., 2014) (UTM, 2021).

Pero a pesar de los esfuerzos realizados por la institución en capacitar continuamente al personal docente, no se ha podido capacitar completamente en estrategias pedagógicas virtuales, que con la finalidad de afrontar las medidas de restricción aún latentes, se ha tratado de adaptar estrategias presenciales a lo virtual como primeros pasos en este complejo proceso, que requiere de la innovación y experiencia del personal docente universitario, ya que la educación en estos niveles tiene mayores exigencias de acuerdo a las competencias y perfiles de egresos (Orellano, 2012).

Así mismo la carencia de adecuadas estrategias pedagógicas, nos enfrenta día a día al cuestionamiento cada vez más creciente del rigor académico de la modalidad en línea, al darse casos de deshonestidad académica en las actividades de aprendizaje autónomo y la forma de responder los test que cierran las unidades programadas.

Con respecto a la deshonestidad académica, este es un fenómeno que altera y afecta el aprendizaje y desempeño académico, el 
proceso de evaluación, las relaciones entre profesor - estudiante y estudiante - estudiante, así como la integridad de la institución y, por lo tanto, la formación en valores personales y grupales (Diez, 2014).

Resaltando que el proceso de evaluación no solo tiene fines de promoción, sino también de formación, y por tanto es importante que los profesores apliquen instrumentos adaptados a la educación en línea que permitan verificar los aprendizajes, identificando las deficiencias del proceso educativo y concentrando los esfuerzos en los aspectos necesarios para lograr su objetivo (C. E. S., 2020).

Siendo la manera más práctica de afrontar la deshonestidad, un mayor compromiso ético, así como la formación en habilidades y capacidades frente a la virtualidad, realizando las adaptaciones y el ajuste en las estrategias metodológicas, tomando las debidas medidas de seguridad que garanticen la honestidad académica en los procesos de evaluación, que respondan a la dinámica de las exigencias de la sociedad, en pro de garantizar la calidad académica (Hurtado, 2020) (C. E. S., 2020).

Por lo tanto, la construcción del conocimiento utilizando las TIC, combinan estrategias didácticas acompañadas de técnicas, como el aprendizaje colaborativo basado en la interacción de sus participantes, construyendo estructuras de comunicación y asumiendo de forma efectiva el rol de mediador dentro de esos ambientes.

Morocho y Rama (2013) consideran que es importante destacar que la educación a distancia en el Ecuador se encuentra en constante crecimiento gracias al impulso de los avances tecnológicos y de las herramientas de la web 2.0, las videoconferencias, entornos virtuales de aprendizaje y otros recursos tecnológicos que han potenciado la interacción y comunicación con los estudiantes a distancia.
Tomando protagonismo las actividades favorecedoras del proceso enseñanza aprendizaje en la asignatura de Farmacología de la carrera de Medicina de la UTM (Ponce, 2021a) en la evaluación del desempeño de los/las estudiantes han sido:

- Test de conocimientos de las unidades impartidas.

- Análisis y comprensión de fuentes bibliográficas y documentales sobre principios terapéuticos aplicados en aparatos y sistemas.

- Manejo de bases de datos y acervos bibliográficos sobre los conceptos más relevantes tratados en clases.

- Reseña descriptiva de los principales fármacos empleados en patologías prevalentes mediante fichaje.

Cada una de estas actividades se basan en el aprendizaje autónomo, mediante el trabajo cooperativo y basado en problemas, sobre todo, en el análisis de los estudios de casos clínicos farmacológicos y las revisiones bibliográficas de la terapéutica basada en la evidencia científica en base al cuadro nacional básico de medicamentos en su décima revisión.

\section{IV.Tendencias y posibilidades en la ense- ñanza y aplicación profesional de la Far- macología}

El propósito de la docencia es el logro de los resultados de aprendizaje para la formación integral de ciudadanos profesionales comprometidos con el servicio, aporte y transformación de su entorno. Se enmarca en un modelo educativo-pedagógico y en la gestión curricular en permanente actualización; orientada por la pertinencia, el reconocimiento de la diversidad, la interculturalidad y el diálogo de saberes (C.E.S, 2019). Al respecto, Rodríguez et al (2015), refieren que las IES tienen grandes retos como son la generación de conocimientos, formación de profesionales integrales, competentes y con valores, oferta de un posgrado más diversificado y vinculado a la investigación, fortalecimiento del proceso de internaciona-

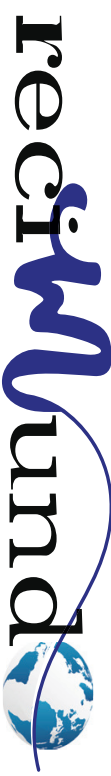


lización, desarrollo de las tecnologías de la información y la comunicación y del sistema de evaluación y participación de la comunidad universitaria en la gestión institucional. De manera que, al integrar el conocimiento de muchas disciplinas científicas relacionadas, en una ciencia como lo constituye la Farmacología, se da la posibilidad de ofrecer una mayor perspectiva en la resolución de problemas, que se asocian con los fármacos, neurotransmisores, hormonas y demás elementos químicos que influyen en la homeostasis orgánica.

Siendo importante señalar que la persona padeciente de una patología crítica presenta cambios fisiopatológicos que inciden en la absorción, distribución, metabolismo y eliminación de los fármacos. Sobre todo, en la hipoperfusión tisular, que afecta la distribución general del fármaco (Carrillo et al., 2013).

Esta situación ya nos ilustra como una condición patológica descompensada puede influir en mayor magnitud en los procesos farmacocinéticos de liberación, absorción, distribución, metabolismo y eliminación (LADME) a partir de las características farmacológicas generales y específicas de un determinado medicamento para conseguir el objetivo terapéutico deseado.

Mientras se continua el desarrollo de nuevos fármacos, para el tratamiento de nuevas enfermedades, y también para mejorar el tratamiento de enfermedades conocidas, sobre todo demostrando mayor efectividad y eficacia, con mínimos efectos secundarios y adversos. Por lo que, en estos nuevos retos terapéuticos, van apareciendo interrogantes que promueven investigaciones clínicas y epidemiológicas, que responden a las necesidades de la población en general. Pero todo este conglomerado de acervos para el desarrollo científico de la Farmacología, requiere de la organización del aprendizaje, a través de las horas y/o créditos, planificados en los siguientes compo- nentes: Aprendizaje en contacto con el docente; aprendizaje autónomo; aprendizaje práctico-experimental (Ponce, 2021a).

Con respecto al último de los componentes que tiene que ver con el aprendizaje experimental, es el entrenamiento clínico constante, el que amplia prospectivamente, el rango de oportunidades laborales en instituciones de salud, en farmacéuticas y en instituciones académicas, que enfrentarán la constante necesidad de la experiencia profesional, en la aplicación de las destrezas adquiridas en el perfil de egreso del futuro profesional de la salud.

Sin pasar por alto, la existencia de una gran diversidad de estrategias y metodologías que pueden aplicarse con éxito para el aprendizaje de la Farmacología pero que requieren un cambio estructural y curricular que apoyen su integración a la asignatura y a la modalidad de impartición (Hidalgo et al., 2017).

En base a esto, la educación a distancia es una modalidad educativa en la que el proceso de enseñanza aprendizaje se caracteriza por la separación del profesor y el estudiante en tiempo y espacio, y se apoya en diferentes recursos educativos que propician y estimulan el aprendizaje autónomo del estudiante y donde existe una institución que garantiza la comunicación multidireccional en el mismo (García et al., 2021).

\section{Criterios para evaluar la educación en línea}

La educación se ampara en un nuevo prototipo que corresponda a los cambios sociales, económicos, políticos, de producción y de desarrollo del conocimiento. Lo cual hace que la calidad y el mejoramiento académico continúen siendo prioridad en la agenda universitaria de intervención (Cevallos y Romero, 2017). 
NAL

De manera que si no se realiza una evaluación de las acciones implementadas, como de los factores que por la emergencia no se consideraron, se corre riesgo de generar acciones educativas "activistas" que prontamente perderán vigencia y sostenibilidad (Crawford et al., 2020).

De acuerdo a lo manifestado por Sánchez y Ruíz (2021), actualmente no es clara la existencia de un modelo de evaluación para las modalidades no presenciales, expedido por el Consejo de Aseguramiento de la Calidad de la Educación Superior (CACES), que permita conocer los estándares, criterios, dimensiones y otros elementos de calidad oficiales que deberían cumplirse en las modalidades en línea. No obstante, distintos organismos y agencias nacionales e internacionales han elaborado instrumentos para evaluar la calidad de la educación en línea, que pueden ser de utilidad para implementarlos en el entorno local.

- Aseguramiento de los mismos resultados de aprendizaje o perfil de egreso de la modalidad presencial, por medio de distintos mecanismos incluyendo simuladores que permitan la adquisición de distintas competencias, especialmente aquellas prácticas o adaptaciones para las personas con necesidades especiales de aprendizaje y la elaboración de guías de estudio que permitan al estudiante con dificultades de acceso a conexión de internet educarse de manera autónoma (C. E. S., 2020)

- Adecuada infraestructura tecnológica, para el desarrollo de las distintas actividades de aprendizaje, incluyendo bibliotecas digitales y el respectivo soporte con el que deben contar las universidades y escuelas politécnicas, como los repositorios digitales de apoyo y los repositorios que contengan las grabaciones de las sesiones de clases ( $C$. E. S., 2020).

- Organización de una estructura de acompañamiento y monitoreo al des- empeño de los estudiantes, así como a brindar servicios de apoyo y asesorías permanentes, para llevar un adecuado sistema de monitoreo o tutoría que propicie y fortalezca la relación enseñanza - aprendizaje (C. E. S., 2020).

- Desarrollo docente de las capacidades digitales y pedagógicas adaptadas a la educación en línea, con respecto al nivel de experiencia y experticia en la educación en línea (C. E. S., 2020).

Quedando pendiente la manera como poder evaluar este proceso educativo virtual impuesto por el contexto de la pandemia por Covid - 19 y adaptado desde la metodología presencial que había sido lo referente en la formación académica desde la creación de las IES nacionales.

\section{Conclusiones}

- La Farmacología es una disciplina elemental que contribuye de gran manera en la formación del futuro profesional de la salud, con la finalidad de mejorar la toma de decisiones y las habilidades clínicas, pudiendo reducir significativamente el error médico en el abordaje terapéutico del Covid - 19.

- La educación a distancia es la protagonista de las metodologías innovadoras, dejando en claro los principios metodológicos y evaluativos del modelo educativo adoptado ante la pandemia por Covid - 19. Cuyo soporte legal reposa en las disposiciones derivadas de la normativa transitoria expedida por el CES, con el objetivo de velar por el derecho a la educación de los/las estudiantes durante la pandemia, sin perder de vista la calidad educativa.

- El mantenimiento de varias actividades educativas, es importante que se planifiquen en base a la complejidad de intereses y los conflictos propios que se suscitan. Todo lo cual, puede incidir en los procesos sustantivos, que deben estar a tiempo en la forma de gestionar

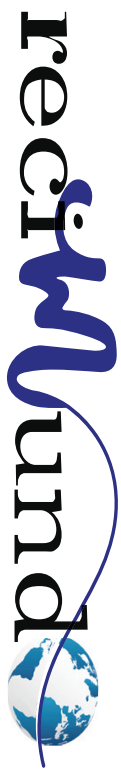


tiempos, insumos y recursos.

- Las practicas virtuales incluyendo las tutorías, han sido retomadas en la mayoría de IES gracias a las condiciones favorables de las prácticas de los modelos no presenciales, para poder afrontar el compromiso formativo de los/las estudiantes de Medicina.

- Finalmente, la educación virtual a pesar de las ventajas que tiene su implementación, también deja entrever que ha sido un proceso acelerado, ensimismado y contradictorio cuando las actividades de aprendizaje no están coordinadas dentro de las funciones sustantivas del ejercicio docente, atropellado en ciertos casos, por la sobrecarga de funciones impuestas por algunos directivos bajo la justificación de carga horaria en la modalidad de teletrabajo.

\section{Bibliografía}

Beltrán, R., Beltrán, A., Robert, G., \& Robert, M. (2016). Apuntes para la concreción de los programas analíticos en la disciplina Preparación para la Defensa en ciencias médicas. Revista Información Científica, 95(6), 967-974.

C. E. S. (2020). Normativa transitoria para el desarrollo de actividades académicas en las Instituciones de Educación Superior, debido al estado de excepción decretado por la emergencia sanitaria ocasionada por la pandemia de COVID-19. Consejo de Educación Superior. RPC-SE-03No.046-2020. Gaceta Oficial. http://gaceta.ces. gob.ec/inicio.html?id_documento=238012

Caballero, N. (2013). El Syllabus. Revista El Tecnológico, 23(1), 22.

Carrillo, R., Zavaleta, M., Álvarez, H., Carrillo, D. M., \& Carrillo, C. A. (2013). La importancia de los parámetros farmacocinéticos y farmacodinámicos en la prescripción de antibióticos. Revista de la Facultad de Medicina (México), 56(3), 5-11.

Cedeño, E., \& Murillo, J. (2020). Entornos virtuales de aprendizaje y su rol innovador en el proceso de enseñanza. ReHuSo: Revista de Ciencias Humanísticas y Sociales, 4(1), 119-127.

C.E.S. (2019). Reglamento de Regimen Académico. Consejo de Educación Superior. Registro Oficial 473 de 23-abril-2019. https://www.ces.gob.ec/ lotaip/Anexos\%20Generales/a3_Reformas/r.r.aca- demico.pdf

Cevallos, N., \& Romero, A. (2017). Mejoramiento de la calidad de la educación superior desde la comparación de estándares. INNOVA Research Journal, 2(7), 13-33. https://doi.org/10.33890/innova. v2.n7.2017.223

Córica, J. (2020). Resistencia docente al cambio: Caracterización y estrategias para un problema no resuelto. RIED. Revista Iberoamericana de Educación a Distancia, 23(2), 255-272. https://doi. org/10.5944/ried.23.2.26578

Crawford, J., Butler, K., Rudolph, J., Malkawi, B., Glowatz, M., Burton, R., Magni, P. A., \& Lam, S. (2020). COVID-19: 20 countries' higher education intra-period digital pedagogy responses. Journal of Applied Learning and Teaching, 3(1), 09-28. https://doi.org/10.37074/jalt.2020.3.1.7

Díaz, M. (2016). El verdadero milagro ecuatoriano (Primera). Publioffset.

Diez, E. (2014). Deshonestidad académica de alumnos y profesores. Su contribución en la desvinculación moral y corrupción social. Revista Sinéctica, 1(1), 1-17.

Failache, E., Katzkowicz, N., \& Machado, A. (2020). La educación en tiempos de pandemia. Y el día después [Blog del Departamento de Economía]. Universidad Católica del Uruguay. https://www.colibri. udelar.edu.uy/jspui/handle/20.500.12008/24008

García, A., Domínguez, G., Torres, A., \& López, A. (2021). La educación a distancia en la era de las TIC. Una visión desde los docentes de una carrera técnica de perfil agropecuario. Revista Cognosis. ISSN 2588-0578, 6(2), 93-110. https://doi. org/10.33936/cognosis.v6i2.3613

Gob.ec. (2008). Constitución de la República del Ecuador 2008. https://www.gob.ec/regulaciones/ constitucion-republica-ecuador-2008

Hidalgo, B., Mayacela, Á., \& Hidalgo, I. (2017). Estrategias didácticas para potenciar el aprendizaje de Farmacología clínica. Revista Habanera de Ciencias Médicas, 16(3), 439-453.

Hurtado, F. (2020). La educación en tiempos de pandemia: Los desafíos de la escuela del siglo XXI. CIEG, 1(1), 176-187.

Morocho, M., \& Rama, C. (2013). La educación a distancia y virtual en Ecuador: Una nueva realidad universitaria (Primera). Universidad Técnica Particular de Loja. https://virtualeduca.org/documentos/observatorio/la_educacion_a_distancia_y_virtual_en_ecuador.pdf 
Orellano, C. (2012). Uso de los espacios virtuales para la docencia en cursos de pregrado de Medicina. Revista Medica Herediana, 23(3), 188-192.

Picón, M. (2020). ¿Es posible la enseñanza virtual? Foro Educacional, 34, 11-34. https://doi.org/: https://doi.org/10.29344/07180772.34.2357

Ponce. (2020). La Farmacología en la educación médica universitaria. RECIMUNDO, 4(4), 129-137. https://doi.org/10.26820/recimundo/4.(4).noviembre.2020.129-137

Ponce, J. (2021a). Programa analítico de la asignatura Farmacología II. Universidad Técnica de Manabí. https://spca.utm.edu.ec/\#!Programas\%20 anal\%C3\%ADticos

Ponce, J. (2021b). Farmacología Cardiovascular: Conceptos generales para la práctica terapéutica. Dominio de las Ciencias, 7(3), 903-923. https://doi. org/10.23857/dc.v7i3.2030

Ponce, J., \& Salazar, G. (2021). La relación docente - estudiante como desafío pendiente en la educación superior. Dominio de las Ciencias, 7(3), 877902. https://doi.org/10.23857/dc.v7i3.2029

Rodríguez, D., De León, L., \& Galarza, J. (2015). Los retos actuales de las instituciones de educación superior en el área de la gestión. Revista Universidad y Sociedad, 7(2), 52-55.

Rojas, N., Pérez, F., Torres, I., \& Peláez, E. (2014). Las aulas virtuales: Una opción para el desarrollo de la Educación Médica. EDUMECENTRO, 6(2), 231-247.

Salguero, M. A. (2009). Planificación curricular y didáctica: Modelos (segunda). Ediciones P.H. https://biblioteca.casadelacultura.gob.ec/cgi-bin/ koha/opac-detail.pl?biblionumber=59895
Sanchez, N., \& Ruiz, D. (2021). Estándares mínimos de calidad de la educación superior en Ecuador durante la pandemia por COVID-19, como medio de protección del derecho a la educación superior. Tsafiqui - Revista Científica en Ciencias Sociales, 16, 7-18. https://doi.org/10.29019/tsafiqui. v12i16.877

Sánchez, N., \& Ruíz, D. (2021). Estándares mínimos de calidad de la educación superior en Ecuador durante la pandemia por COVID-19, como medio de protección del derecho a la educación superior. Tsafiqui - Revista Científica en Ciencias Sociales, 16, 7-18. https://doi.org/10.29019/tsafiqui. v12i 16.877

Umaña, A. (2020). Educación Superior en Tiempos de COVID-19: Oportunidades y retos de la educación a distancia. Innovaciones Educativas, 22(Especial), 36-49. https://doi.org/10.22458/ie.v22iEspecial.3199

UNESCO. (2020). La UNESCO y crisis educativa. [ONG]. UNESCO. https://es.unesco.org/ news/290-millones-estudiantes-clases-covid-19-unesco-divulga-primeras-cifras-mundiales-y-se-moviliza

UTM. (2019). Rendición de cuentas 2019. https:// emserving-ep.gob.ec/transparencia/rendicion-de-cuentas-2019/category/2-transparencia

UTM. (2021). Teletrabajo UTM [Institución educativa]. Universidad Técnica de Manabí. https://www. utm.edu.ec/teletrabajo/

Varguillas, C., \& Bravo, P. (2020). Virtualidad como herramienta de apoyo a la presencialidad: Análisis desde la mirada estudiantil. Revista de Ciencias Sociales (Ve), XXVI(1), 219-232.

Vera, O. (2014). Enseñanza de la farmacología basada en competencias. Cuadernos Hospital de Clínicas, 55(4), 43-54.

Zambrano, J., Laurencio, A., López, A., \& Estrada, O. (2018). Fundamentos del modelo para virtualización de la formación en la Universidad Técnica de Manabí, Ecuador: Atenas, 4(44), 78-91.

\section{CITAR ESTE ARTICULO:}

Ponce Alencastro, J. A. (2021). La Farmacología en tiempos de Covid - 19: Desafíos de su enseñanza y aplicación profesional. RECIMUNDO, 5(1 (Suple), 149-163. https://doi.org/10.26820/recimundo/5.(Suple1).oct.2021.149-163

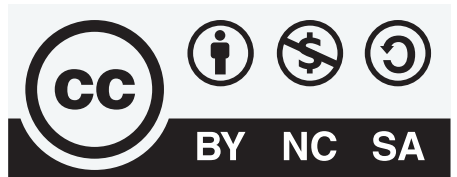

CREATIVE COMMONS RECONOCIMIENTO-NOCOMERCIAL-COMPARTIRIGUAL 4.0. 